\title{
Food photography I: the perception of food portion size from photographs
}

\author{
BY M. NELSON, M. ATKINSON AND S. DARBYSHIRE \\ Department of Nutrition and Dietetics, King's College London, Campden Hill Road, \\ London W8 $7 A H$
}

(Received 8 October 1993 - Revised 11 January 1994 - Accepted 15 February 1994)

\begin{abstract}
Fifty-one male and female volunteers aged 18-90 years from a wide variety of social and occupational backgrounds completed 7284 assessments of portion size in relation to food photographs. Subjects were shown six portion sizes (two small, two medium and two large) for each of six foods, and asked to compare the amount on the plate in front of them to (a) a series of eight photographs showing weights of portions from the 5th to the 95th centile of portion size (British Adult Dietary Survey), or (b) a single photograph of the average (median) portion size. Photographs were prepared either in colour or in black and white, and in two different sizes. The order of presentation of foods; use of black and white or colour; the size of photographs; and presentation of eight or average photographs were each randomized independently. On average, the mean differences between the portion size presented and the estimate of portion size using the photographs varied from -8 to $+6 \mathrm{~g}(-4$ to $+5 \%)$ for the series of eight photographs, and from -34 to $-1 \mathrm{~g}(-23$ to $+9 \%)$ for the single average photograph. Large portion sizes tended to be underestimated more than medium or small portion sizes, especially when using the average photograph (from -79 to $-14 \mathrm{~g},-37$ to $-13 \%$ ). Being female, 65 years and over, or retired, or seeing photographs in colour, were all associated with small but statistically significant overestimations of portion size. Having a body mass index $\geqslant 30 \mathrm{~kg} / \mathrm{m}^{2}$ was associated with an $8 \%$ underestimate of portion size. We conclude that use of a series of eight photographs is associated with relatively small errors in portion size perception, whereas use of an average photograph is consistently associated with substantial underestimation across a variety of foods.
\end{abstract}

Food photography: Dietary survey: Epidemiology

Photographs of food have often been used in dietary surveys to help subjects estimate portion size. Typically, photographs are taken of small, medium and large portions which are judged to be representative of the range of portion sizes actually consumed. Subjects are then asked to identify which photograph best reflects either their usual portion size (e.g. in a diet history) or actual portion size (e.g. in a $24 \mathrm{~h}$ recall). Alternatively, a single photograph of average portion size is displayed, and subjects are asked to estimate their own portion size as a fraction, multiple or percentage of the amount shown in the photograph.

A complex process takes place when a photograph is used to identify portion size during an interview or questionnaire completion. This process has three main elements: perception, conceptualization, and memory. Perception involves a subject's ability to relate an amount of food which is present in reality to an amount depicted in a photograph. Conceptualization concerns a subject's ability to make a mental construct of an amount of food which is not present in reality, and to relate that to a photograph. Memory will affect the precision of the conceptualization.

A number of studies have examined portion size assessment and the use of photographs. Guthrie (1984) reported that between 14 and $67 \%$ of assessments of food portion size were 
in error by more than $50 \%$ when no aid was used. Pietinen and co-workers $(1988 a, b)$ compared estimates of nutrient intake from weighed records with those from food frequency and amount questionnaires (FAQ) administered either with or without photographs. The use of photographs improved the level of agreement between the FAQ and the weighed records. Whereas some studies have used a set of photographs for each food depicting several portion sizes (Chu et al. 1984; Pietinen et al. 1988 a; Edington et al. 1989; Hankin et al. 1991; Tjonneland et al. 1991), others have used only a single photograph (Byers et al. 1985; Irish National Food Survey, 1990) while others still have used photographs in conjunction with other aids (e.g. models, cups) (Rutishauser, 1982; Samet et al. 1984). The diversity of methods in these studies makes it difficult to compare outcomes regarding the value of different approaches to portion size assessment.

The general conclusion from these studies is that photographs are of benefit in helping subjects to assess portion size. It is inevitable, however, that inaccuracies in portion size assessment will remain. These errors will lead to misclassification of subjects according to the amount of food consumed or the level of nutrient intake. The degree of misclassification can be reduced only if the components of the error are fully described.

There is no research to date which looks specifically at the errors associated with each of the elements of the process involved in estimating food portion sizes from photographs. This paper reports for the first time the errors associated with perception. It examines these errors in relation to factors which are potential influences on perception, such as the size and number of photographs displayed or whether the photographs are in black and white or colour. It also looks at factors relating to the subjects themselves (sex, age, body size) and to the type and amount of food being assessed.

\section{METHODS}

Sample

The aim was to obtain a good cross-section of men and women aged between 18 and 90 years from a wide variety of social and occupational backgrounds. Table 1 shows the sample composition. Recruitment of employed subjects was mainly through local employers and the local authority, and for subjects retired or not employed, through voluntary groups and day centres. All subjects were volunteers.

\section{Choice of foods}

Six commonly eaten foods were chosen. They excluded foods which are easy to describe in household measures (e.g. slices of bread, biscuits, eggs). The aim was to include foods for which some aid to portion size assessment would be necessary in an interview or questionnaire, and to span selected characteristics of appearance which were likely to influence perception of amounts from photographs: area and depth of pieces or mounds on a plate; number and size of pieces; area and thickness of slices; and depth in a bowl. The six foods chosen and their main characteristics for assessment in relation to perception were: (1) boiled potato, number and size of pieces; (2) mashed potato, depth and area of a mound of dry, stiff food; (3) quiche, area and depth of triangular slice; (4) cornflakes, depth of mound in bowl; (5) roast carcass meat, area, thickness and number of slices; (6) spaghetti, depth and area of serving of 'slippery' food.

\section{Portion sizes in photographs}

Portion size data were derived from the British Adult Dietary Survey (Gregory et al. 1990). For each food the distribution of reported food portion or serving weights was used to determine portion weights corresponding to the 5 th, 50 th and 95 th centiles. 
Table 1. Occupation and age group of fifty-one subjects completing measurements of perception of food portion size from photographs

\begin{tabular}{|c|c|c|c|c|c|}
\hline \multirow[b]{2}{*}{ Occupation... } & \multicolumn{2}{|c|}{ Men } & \multicolumn{3}{|c|}{ Women } \\
\hline & Manual & Non-manual & Manual & Non-manual & Non-working* \\
\hline \multicolumn{6}{|c|}{ Age group (years) } \\
\hline $18-29$ & 3 & 3 & 2 & 3 & 2 \\
\hline $30-44$ & 3 & 3 & 2 & 4 & 2 \\
\hline $45-64$ & 3 & 3 & 2 & 2 & 2 \\
\hline Over 64 & \multirow{2}{*}{\multicolumn{2}{|c|}{6}} & \multirow{2}{*}{\multicolumn{2}{|c|}{6}} & \\
\hline Total & & & & 27 & \\
\hline
\end{tabular}

*Non-working women are those not currently in paid employment.

Nine photographs were taken for each food. One photograph (referred to as the 'Average') showed portion weight at the 50th centile. Eight photographs were taken showing portion sizes between the 5 th and 95 th centiles, one each at the 5 th and 95 th centiles, and the remaining six photographs at equal intervals between these two portion sizes. The values for the weights of food shown in the photographs are shown in the Appendix.

\section{Preparation of photographs}

Photographs were taken under standard lighting conditions in a professional studio. All foods except cornflakes were photographed on a white $10^{\prime \prime}$ diameter dinner plate with a dinner knife to the right of the plate and a dinner fork to the left against a white background. Cornflakes were photographed in a white 6 " diameter bowl with a dessert spoon to the right of the bowl. Contrast between the white plate or bowl and the white background was achieved through lighting effects. All photographs were taken from an angle of $42^{\circ}$ above the horizontal, which was assessed as an average angle of viewing for a subject seated at a dining table.

All photographs were printed in the landscape orientation in both black and white and in colour. The 'Average' photographs were printed in two sizes, A5 (approximately $150 \times 200 \mathrm{~mm}$ ) and A6 (approximately $100 \times 150 \mathrm{~mm}$ ). The series of eight photographs was also printed in two sizes, A6 and A7 (approximately $75 \times 100 \mathrm{~mm}$ ).

\section{Portion sizes for assessment}

Six portions were prepared for each food. Two different portions were prepared in each of three size intervals: small (food weight between the weights shown in the first and third photograph in the series of eight); medium (food weight between the third and sixth photograph); and large (food weight between the sixth and eighth photograph). The weights of the foods presented to the subjects are shown as two series (A and B) in Table 2 . The two series relate to the randomization carried out during the assessment procedure (see below). The portion sizes selected for boiled and mashed potato were identical.

\section{Assessment procedure}

Subjects were invited in groups of four to attend the Nutrition Department at King's College London for two $2 \mathrm{~h}$ sessions. At the first session each subject completed a short 
Table 2. Food portion sizes ( $\mathrm{g}$ ) presented to subjects during the course of assessment of perception*

(Values for categories show the range of food weights in the photographs: small, photographs 1-3; medium, photographs 3-6; large, photographs 6-8 (see Appendix))

\begin{tabular}{|c|c|c|c|}
\hline \multirow[b]{2}{*}{ Food } & \multicolumn{3}{|c|}{ Portion wt (g) } \\
\hline & Category & Portion A & Portion B \\
\hline \multirow[t]{3}{*}{ Mashed potato } & Small 58-139 & 87 & 107 \\
\hline & Medium 140-259 & 243 & 202 \\
\hline & Large $260-345$ & 271 & 322 \\
\hline \multirow[t]{3}{*}{ Boiled potato } & Small 58-139 & 90 & 63 \\
\hline & Medium $140-259$ & 212 & 176 \\
\hline & Large $260-345$ & 322 & 287 \\
\hline \multirow[t]{3}{*}{ Quiche } & Small $62-105$ & 88 & 86 \\
\hline & Medium 106-169 & 136 & 164 \\
\hline & Large $170-218$ & 178 & 200 \\
\hline \multirow[t]{3}{*}{ Cornflakes } & Small 16-32 & 21 & 27 \\
\hline & Medium 33-59 & 49 & 41 \\
\hline & Large $60-77$ & 66 & 74 \\
\hline \multirow[t]{3}{*}{ Spaghetti } & Small 54-159 & 80 & 86 \\
\hline & Medium $160-319$ & 202 & 317 \\
\hline & Large $320-425$ & 403 & 357 \\
\hline \multirow[t]{3}{*}{ Sliced meat } & Small 26-69 & 53 & 37 \\
\hline & Medium $70-139$ & 99 & 82 \\
\hline & Large $140-190$ & 189 & 178 \\
\hline
\end{tabular}

* For details of subjects and procedures, see Table 1 and pp. 650-653.

questionnaire providing personal details on age, sex, height, weight, occupation, colour blindness, and weight gain or weight loss in the previous year. Working in a taste panel room in which subjects were screened from one another, each subject was presented with a plate or bowl of one food in one of the six portion sizes given in Table 2, together with the photograph(s) of that food. The plate or bowl was identical to that in the photographs. In any one session, subjects evaluated foods either in relation to the sets of eight photographs or in relation to the average photographs. The eight photographs were presented on a single sheet and numbered from ' 1 ' (smallest) to ' 8 ' (largest).

Within any one session, subjects saw either single average photographs only or the series of eight photographs only. Within any half session, subjects saw either black and white photographs only or colour photographs only. The order of presentation of the series of eight or single average photographs and black and white or colour photographs was randomized between sessions. Four randomized series of order of presentation regarding size of photograph, food, and portion size were generated. Within each half session the same randomized sequence was used for every subject. The randomization therefore included eight $v$. average photographs, black and white $v$. colour, foods, portion size, and size of photograph, and each of these contrasts was treated as independent for the statistical analyses.

Each half session lasted not more than $45 \mathrm{~min}$, in which each subject completed thirtysix assessments. Subjects had a short tea break between half-sessions. At the end of both $2 \mathrm{~h}$ sessions, each subject had completed 144 assessments. A total of $7344(51 \times 144)$ assessments was planned.

At the start of each half session involving the use of series of eight photographs, subjects were given a sheaf of six pages stapled together. Six plate numbers in the randomized 
sequence were listed on each page, each of which was headed with the following instruction: 'The points 1 to 8 on the scale represent the eight photographs of portion size presented for each food. Please mark a cross at any position on the line which you think most closely represents the portion size of the food presented.' Foods were presented in the order in which they were listed on the page. Subjects were presented with an $11.5 \mathrm{~cm}$ visual analogue scale (VAS) as shown below:

\section{PLATE NUMBER}

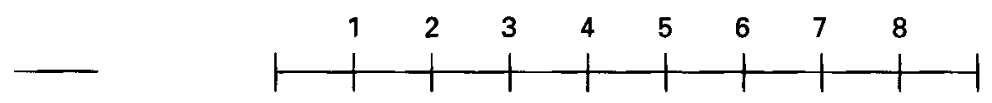

Each food was passed through a serving hatch on a numbered plate with the corresponding photographs, and the subject was asked to place a cross at the point on the line according to the instruction above.

For the average photographs, subjects were given two stapled sheets in each half session and, again, foods were presented in the order in which they were listed on the page. Subjects were asked to write down a number which expressed the amount on the plate as a fraction, multiple or percentage of the amount shown in the photograph. After assessing each food portion, the plate was handed back through the serving hatch, and the next food and photograph(s) were presented.

\section{Statistical analysis}

Data were analysed using the SPSSX computer program on a Digital VAX computer. For each assessment the weight of the food actually presented was subtracted from the weight estimated from the VAS (for the eight photographs) or the fraction or multiple (of the average photograph). Thus, a positive value indicates an overestimate of the weight, and a negative value an underestimate. The percentage difference was the difference in grams divided by the weight of the food portion presented multiplied by 100 .

Differences between estimated and actual food portion sizes were assessed using the paired $t$ test. Statistical analysis of the differences in the error of estimate between colour $v$. black-and-white, eight photographs $v$. average photograph, portion size effect, and the other variables, were carried out using analysis of variance with food as the covariate.

The study was approved by the King's College Human Experimentation Committee.

\section{RESULTS}

Of the 7344 assessments, $160(2 \cdot 2 \%)$ were not usable, either because there were two or more marks on the VAS used with the eight photographs, or because the fraction, multiple or percentage indicated (e.g. ' $-400 \%$ less') could not be sensibly interpreted.

Table 3 shows for each food the mean difference between estimated and actual weight, the mean percentage difference, and the rank correlation between estimated and actual weights. Portion size tended to be underestimated rather than overestimated, significantly more so using the average photograph than the eight photographs. Because of the very large number of observations, the estimated weights were statistically significantly different from the actual weights (paired $t$ test, $P<0.01$ ) for every food except for cornflakes when estimated using the average photograph. Similarly, the percentage differences were statistically significantly different from zero with the exceptions of mashed potato, cornflakes and sliced meat (for eight photographs) and quiche and spaghetti (for the 
M. NELSON AND OTHERS

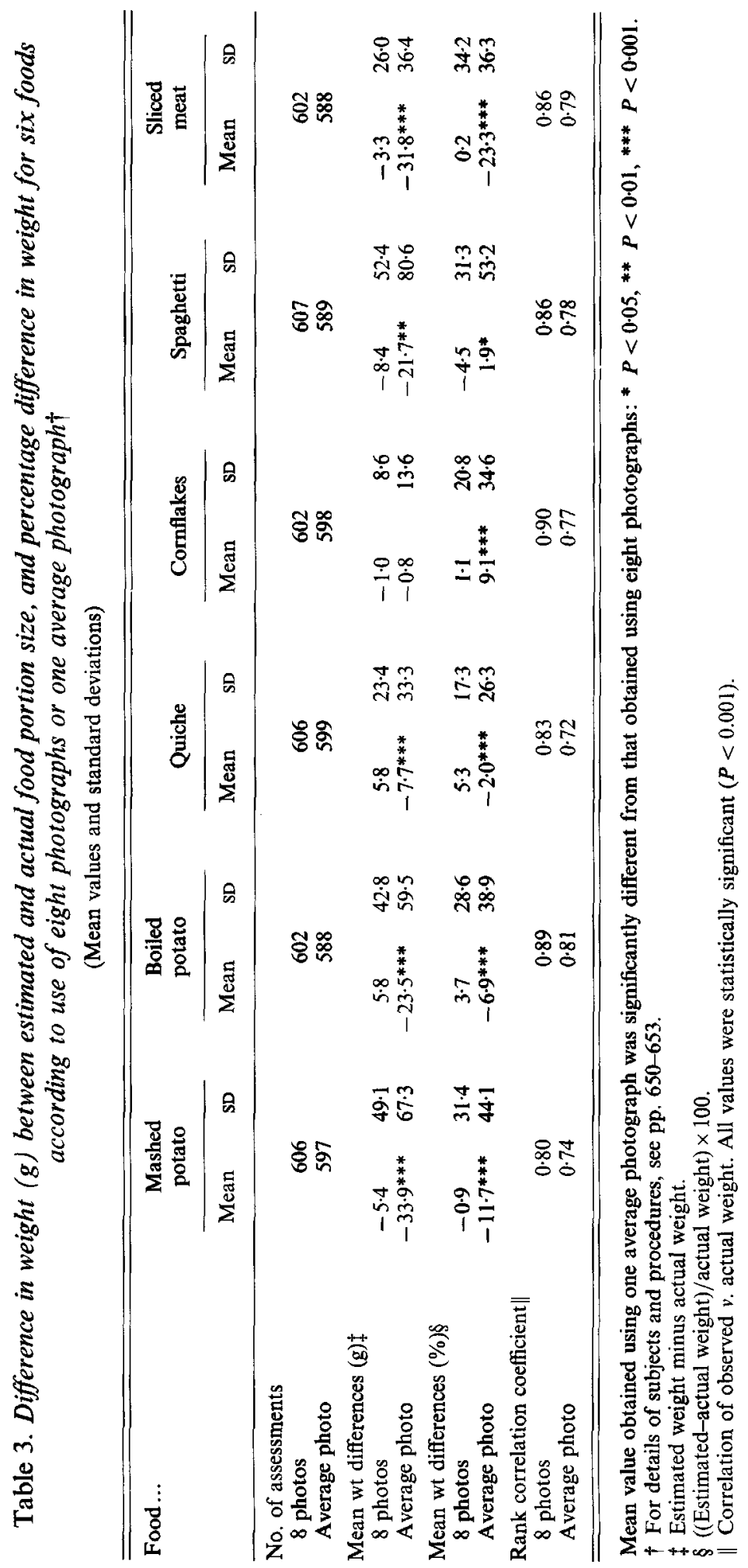


FOOD PORTION SIZE PERCEPTION FROM PHOTOGRAPHS

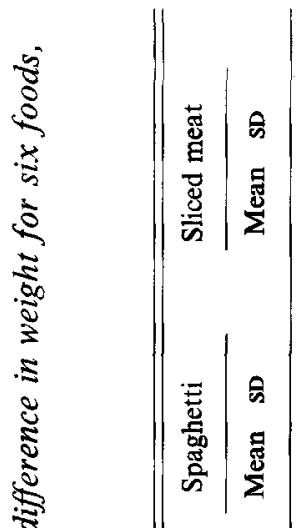

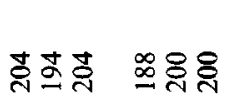

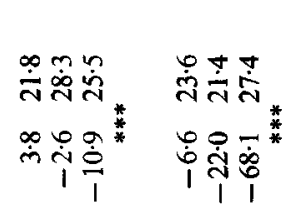

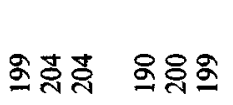

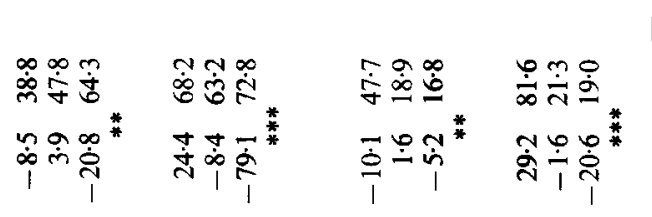

高

(a)

$\stackrel{8}{8}$

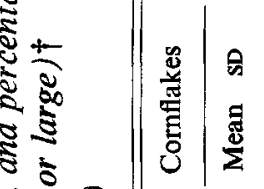

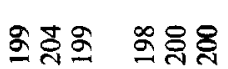

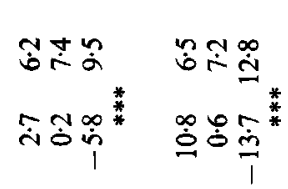

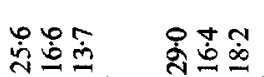

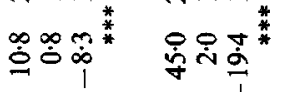

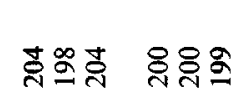

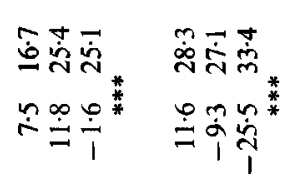

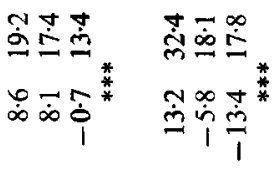

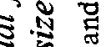

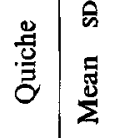

ช.

క

웡

空

웜유 웜

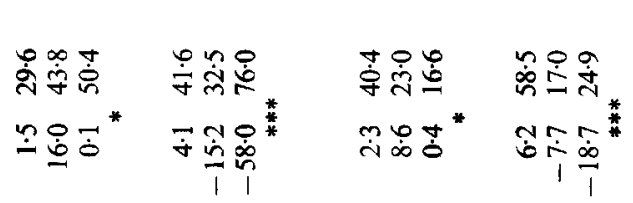

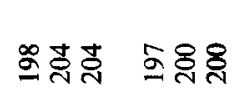

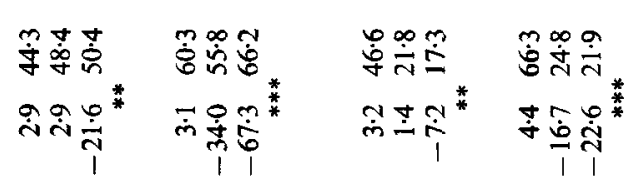

:

$\Xi$

ะั

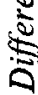

迥

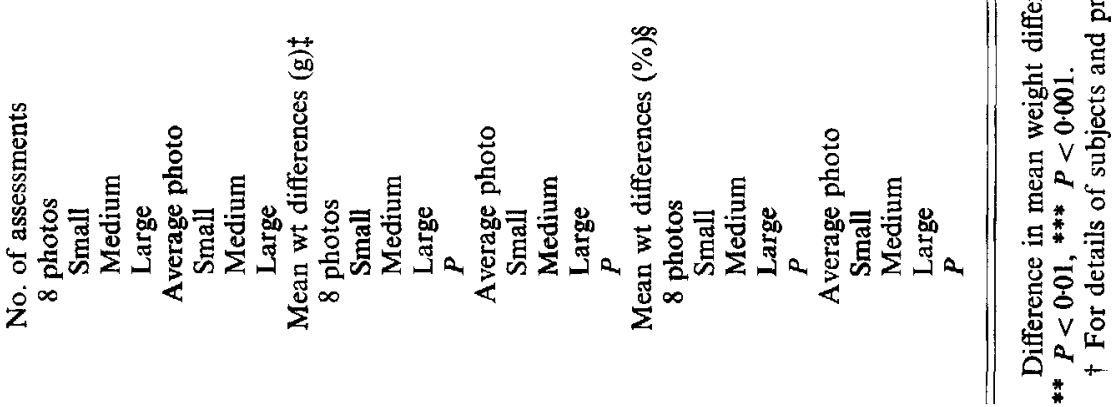



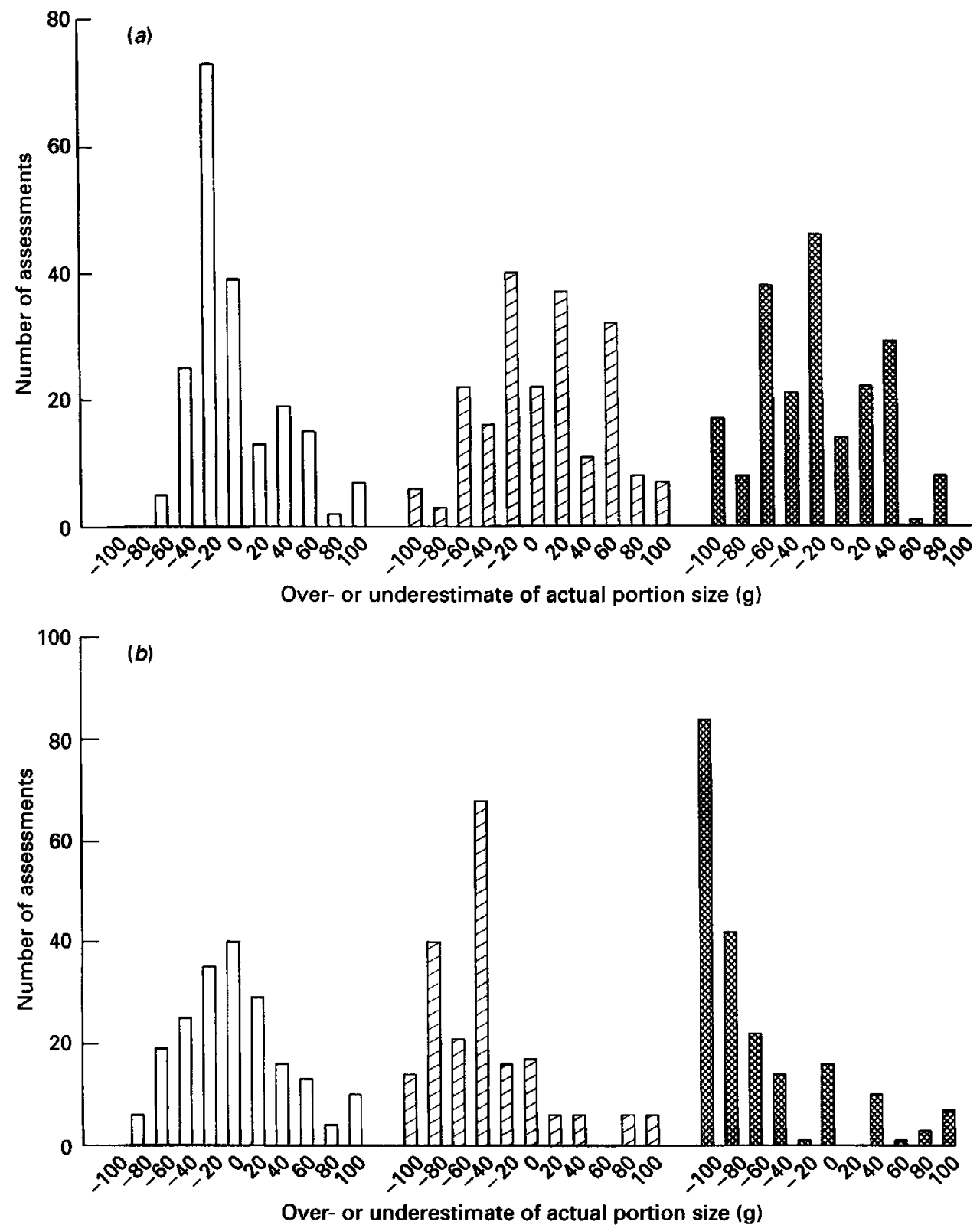

Fig. 1. Differences between actual portion weights of mashed potato and estimates obtained using (a) eight photographs and $(b)$ average photographs, by portion size. ( $\square$ ), small portion; ( $₫)$, medium portion; (因), large portion.

average photographs). The correlation coefficients show that the estimated portion sizes were ranked more closely to the actual weights using the eight photographs than using the average photograph. The apparent discrepancy between the negative mean weight differences and the positive mean percentage weight differences for cornflakes, spaghetti and sliced meat is a mathematical artifact which is resolved when differences in portion size are taken into account.

Table 4 gives results as for Table 3 shown according to portion size. The large portions 


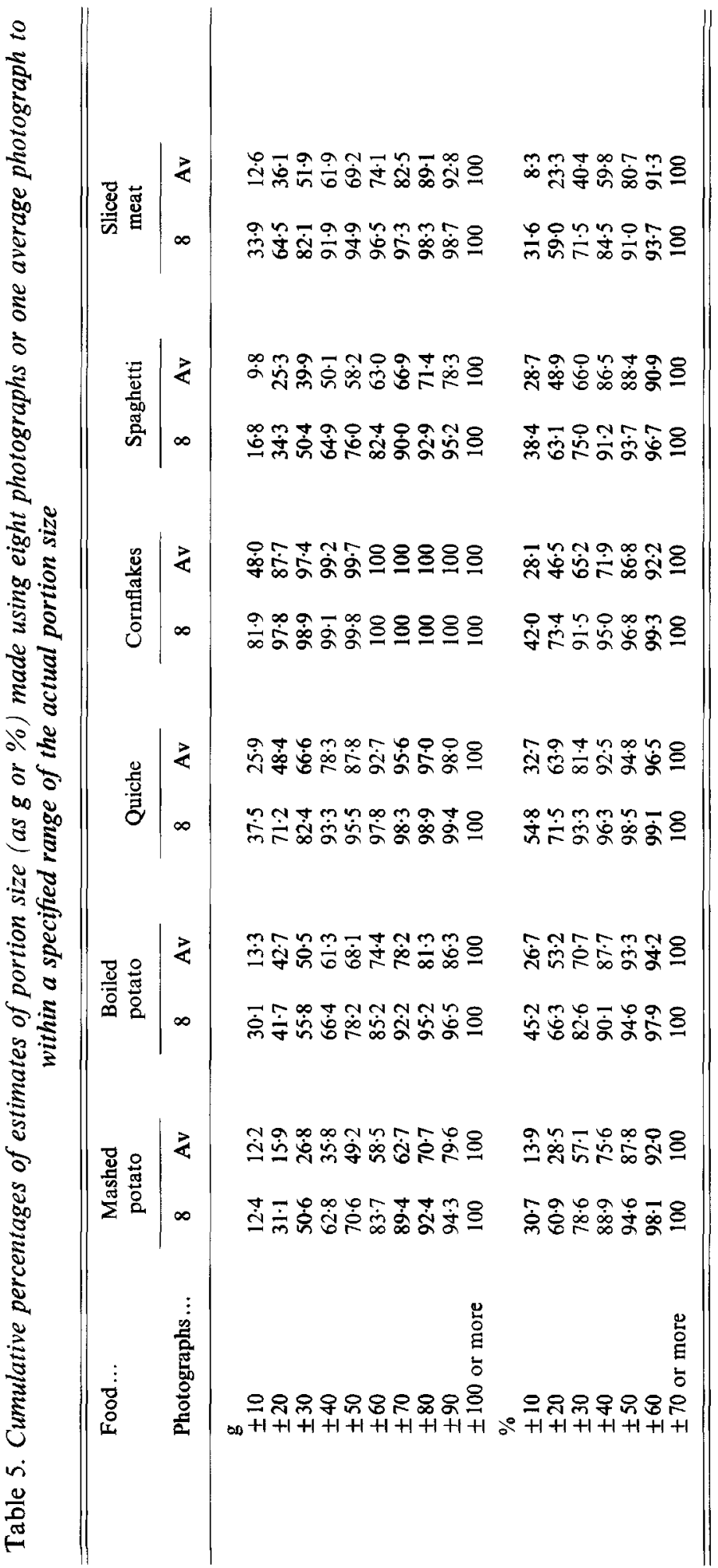


Table 6. Mean percentage differences between estimated and actual portion weights, by sex, age group, occupational group, and body size group, for assessments using eight photographs or one average photograph*†

(Mean values and standard deviations)

\begin{tabular}{|c|c|c|c|c|c|}
\hline \multirow[b]{2}{*}{ Variable } & \multirow[b]{2}{*}{$n$} & \multicolumn{2}{|c|}{ Eight photographs } & \multicolumn{2}{|c|}{ Average photograph } \\
\hline & & Mean & SD & Mean & SD \\
\hline \multicolumn{6}{|l|}{ Sex } \\
\hline Male & 24 & -1.4 & $27 \cdot 1$ & -8.9 & 38.8 \\
\hline Female & 27 & 2.8 & $28 \cdot 8$ & -2.5 & 42.6 \\
\hline \multicolumn{6}{|l|}{ Age group (years) } \\
\hline $18-29$ & 12 & 1.0 & $26 \cdot 4$ & $-6 \cdot 9$ & $42 \cdot 8$ \\
\hline $30-44$ & 16 & $-2 \cdot 0$ & $26 \cdot 8$ & -7.8 & $38 \cdot 2$ \\
\hline $45-64$ & 11 & $-2 \cdot 0$ & $24 \cdot 6$ & $-5 \cdot 1$ & $39 \cdot 1$ \\
\hline $65+$ & 12 & 7.0 & $33 \cdot 1$ & $-1 \cdot 1$ & $44 \cdot 0$ \\
\hline \multicolumn{6}{|c|}{ Body mass index $\left(\mathrm{kg} / \mathrm{m}^{2}\right)$} \\
\hline$<25$ & 27 & 0.9 & 27.8 & $-8 \cdot 3$ & $36 \cdot 7$ \\
\hline $25-29.9$ & 20 & $2 \cdot 4$ & $29 \cdot 2$ & -1.0 & $44 \cdot 4$ \\
\hline $30+$ & 4 & -7.5 & $23 \cdot 1$ & $-7 \cdot 1$ & $49 \cdot 3$ \\
\hline \multicolumn{6}{|l|}{ Occupation group } \\
\hline Non-manual & 20 & -0.8 & $22 \cdot 7$ & $-8 \cdot 7$ & $37 \cdot 4$ \\
\hline Manual & 16 & -0.8 & $26 \cdot 3$ & $-5 \cdot 8$ & 39.8 \\
\hline $\begin{array}{l}\text { Unemployed or } \\
\text { not working }\end{array}$ & 6 & $3 \cdot 0$ & $35 \cdot 0$ & $-1 \cdot 8$ & $46 \cdot 2$ \\
\hline Retired & 9 & $6 \cdot 0$ & $35 \cdot 4$ & $0 \cdot 1$ & $46 \cdot 1$ \\
\hline
\end{tabular}

* For details of subjects and procedures, see pp. 650-653.

$\uparrow$ All analyses of variance between variables controlling for food and portion size, by eight photographs or average photograph, $P<0.01$.

were consistently underestimated, with the exception of boiled potato using eight photographs. The underestimates were most pronounced for the average photograph, where the large portion size was underestimated on average from $14 \mathrm{~g}$ (for cornflakes) to as much as $79 \mathrm{~g}$ (for spaghetti), or in percentage terms, from $13 \%$ (for quiche) to $37 \%$ (for sliced meat). For every food the size of the portion had a statistically significant effect on the amount of under- or overestimation of portion weight, although the degree of underor overestimation was consistently much lower when using the eight photographs than when using the average photograph. The range of variation, reflected in the standard deviation, was generally greater for the average photograph than for the eight photographs.

The typical range of variation is shown in Fig. 1, which plots for each assessment of mashed potato the under- or overestimate of portion weight according to portion size using eight photographs or the average photograph. Using eight photographs, $71 \%$ of assessments were within $50 \mathrm{~g}$ of the actual weight. The errors were smaller for the small portion and larger for the large portion. In contrast, only $49 \%$ of the assessments using the average photograph were within $50 \mathrm{~g}$ of the actual weight, and for the large portion, only $20 \%$ were within $50 \mathrm{~g}$, the majority ( $74 \%$ ) being more than $50 \mathrm{~g}$ below the actual weight. For all foods, estimated portion sizes were within $\pm 20 \%$ of the actual weight for between $59 \%$ (for sliced meat) and $73 \%$ (for cornflakes) of observations when using eight photographs. With the average photographs, between $23 \%$ (for sliced meat) and $64 \%$ (for quiche) of estimates were correct to within $\pm 20 \%$. The details of the number of observations correctly classified to within a specified range are given in Table 5 .

In dietary surveys, sex, age, body size and occupation are potential confounding 
Table 7. Mean percentage differences between estimated and actual portion weights, by black and white or colour photographs, and by size of photograph, for assessments using eight photographs or one average photograph*

(Mean values and standard deviations)

\begin{tabular}{|c|c|c|c|c|}
\hline \multirow[b]{2}{*}{ Variable } & \multicolumn{2}{|c|}{ Eight photographs } & \multicolumn{2}{|c|}{ Average photograph } \\
\hline & Mean & SD & Mean & SD \\
\hline \multicolumn{5}{|c|}{ Black-and-white or colour $\dagger$} \\
\hline Black-and-white & -0.2 & $26 \cdot 5$ & -6.6 & $43 \cdot 3$ \\
\hline Colour & 1.9 & $29 \cdot 6$ & $-4 \cdot 3$ & $38 \cdot 6$ \\
\hline \multicolumn{5}{|l|}{ Size of photograph } \\
\hline A5 $(150 \times 200 \mathrm{~mm})$ & \multicolumn{2}{|c|}{-} & $-4 \cdot 9$ & $45 \cdot 2$ \\
\hline A6 $(100 \times 150 \mathrm{~mm})$ & $1 \cdot 2$ & $29 \cdot 4$ & $-6 \cdot 0$ & $36 \cdot \overline{5}$ \\
\hline A7 $(75 \times 100 \mathrm{~mm})$ & 0.4 & $26 \cdot 8$ & \multicolumn{2}{|c|}{-} \\
\hline
\end{tabular}

* For details of subjects and procedures, see pp. 650-653.

$\dagger$ Analyses of variance between variables controlling for food or portion size, by eight photographs or average photograph, $P<0.01$.

variables. The mean percentage differences in weight across all foods and portion sizes in relation to eight or average photographs are shown for these variables in Table 6 . The observed differences between males and females, age groups, body mass index (BMI) groups and occupational groups are highly statistically significant $(P<0.01$; analysis of variance controlling for food and portion size). Males underestimated portion sizes compared with females, the difference in the error being greater for the average photographs than the eight photographs. The $65+$ age group tended to overestimate portion size for the eight photographs, and underestimate less than the other groups using the average photographs. Subjects with high BMI $\left(\geqslant 30 \mathrm{~kg} / \mathrm{m}^{2}\right)$ underestimated portion size compared with the other groups when using eight photographs. Being retired was associated with overestimating portion size, but this effect disappeared when age was taken into account.

Table 7 shows the effects of black and white $v$. colour photographs, and different photograph size on the estimates of portion size. Colour photographs were associated with a slight overestimation using the eight photographs, and with less underestimation using the average photographs (analysis of variance controlling for food and portion size, $P<0.01$ ). Small differences in the mean percentage difference using different size photographs were not statistically significant.

\section{DISCUSSION}

The results from this study point to clear and consistent biases in the perception of food portion size from photographs. Specifically: (1) use of single or average photographs is associated with much larger errors in estimate of portion size than use of a series of eight photographs; and (2) large portions are likely to be underestimated, more so using average photographs than eight photographs.

The subjects were all volunteers who knew of the general nature of the study at the outset. While they cannot be said to be representative of the population as a whole, they do represent a good cross-section of people from all walks of life. There is no reason to believe that the subjects are different with regard to their perception of food from photographs than other members of the population. 


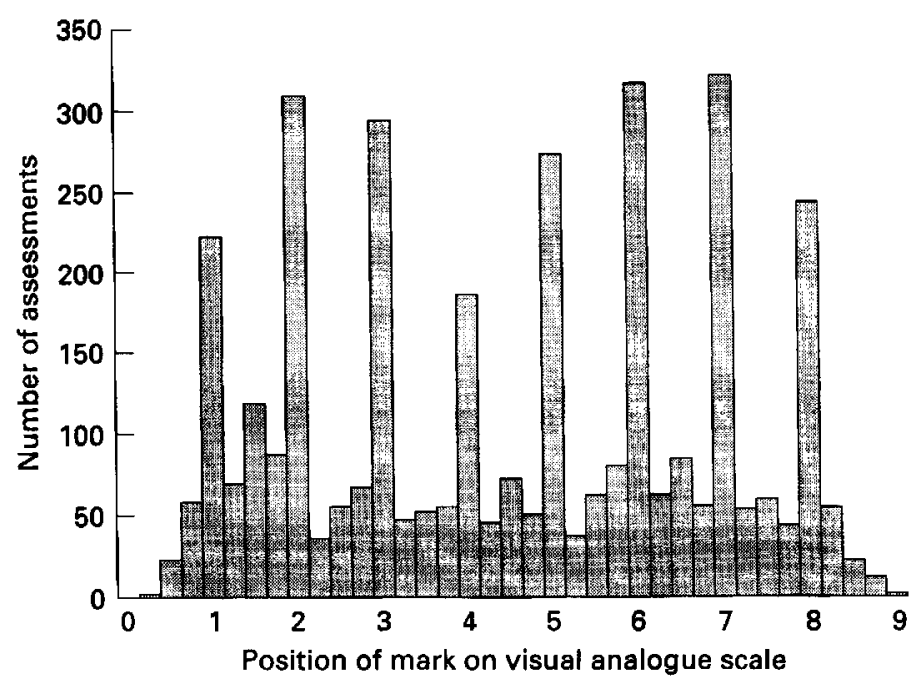

Fig. 2. Position of the mark placed by subjects on a visual analogue scale in relation to eight photographs for all six foods ( $n 3625$ assessments).

The majority $(97 \cdot 8 \%)$ of the assessments were completed successfully. There was no single food or set of assessments which was more problematic than another, although the subjects reported having more difficulty in assessing amounts as a fraction or multiple of the average photograph than in using the VAS. Many subjects reported a progressive awareness of the factors which might influence their perception of portion size such as thickness of slices or size of pieces, but there were no obvious learning or fatigue effects.

The underestimate of portion size using the average photograph shown in Table 3 is explained largely by the underestimate associated with the medium and large portion sizes (Table 4). For all six foods the size of the error for the large portions (both in absolute and percentage terms) was at least three to four times as great using the average photographs compared with the eight photographs. Moreover, the spread of errors (as shown by the standard deviations) was wider using the average photographs.

The largest errors occurred for mashed potato and spaghetti, and the smallest for cornflakes. Subjects found it difficult to estimate the depth of the mashed potato and spaghetti in the photographs. This problem would have been partially resolved by reducing the camera angle to make the depth more apparent. This would then have reduced the ease with which the area of the food on the plate could be assessed, and may have been counter-productive. With cornflakes, subjects stated that an important clue to the amount in the bowl was the width of the exposed area on the side of the bowl not covered by the cereal. With the cornflakes (and the other foods also) the error in perception may have been greater if subjects had had food served on different crockery.

There is some evidence of the 'flat-slope' syndrome. Small portion sizes tended to be overestimated, and large portion sizes underestimated. Again, the error was much greater using the average than the eight photographs.

Part of the error was associated with the measuring devices themselves. Although subjects were told that when using the VAS they could put a mark anywhere on the line, there was a tendency to put a mark corresponding to one particular photograph (Fig. 2). Comparison of the values in Table 2 (weights of portions as presented) and the Appendix (weights of foods in photographs) shows that only accasionally were they within $1-2 \mathrm{~g}$. Given this tendency, it suggests that the size of the error in the estimate would be that much larger if only three portion sizes were presented (e.g. in studies by Pietinen et al. $1988 a$ and 


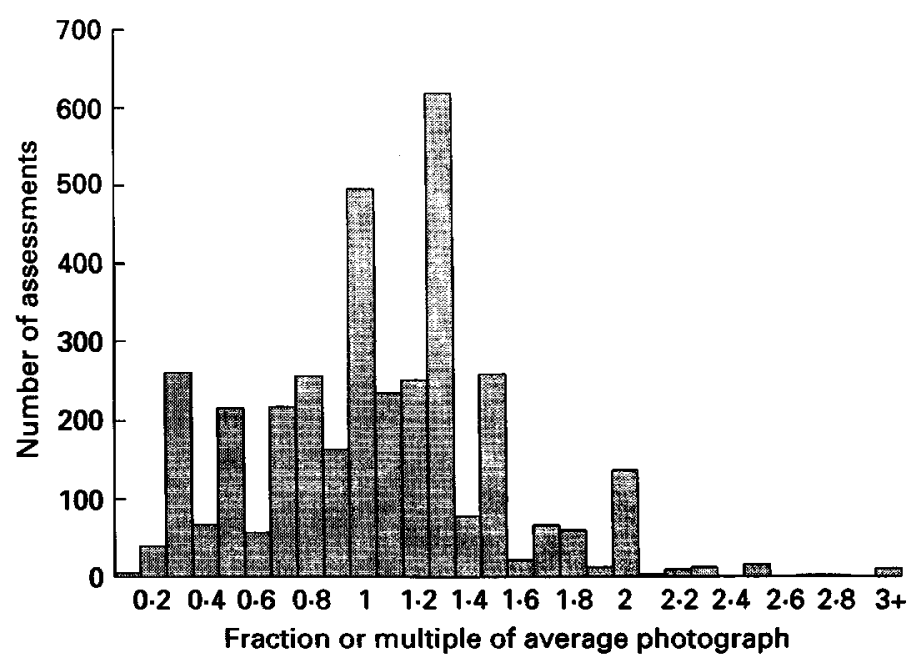

Fig. 3. Fraction or multiple of the average photograph chosen by subjects to describe given portions of six foods ( $n 3559$ assessments).

Hankin et al. 1991). A future study will assess the size of the errors associated with the use of either four or eight photographs.

A similar picture emerges regarding the use of the average photographs. Fig. 3 shows that the subjects tended to use convenient fractions $(1 / 4,1 / 3,1 / 2,3 / 4)$ or multiples (e.g. $1,1 \cdot 25,1 \cdot 5)$ or percentages ( $75 \%$ less, $120 \%, 25 \%$ more). There was a clear reluctance to use multiples larger than 1.5 , in spite of the fact that many of the large portions were twice the amount depicted in the photograph. The size of the error associated with the use of average photographs is therefore likely to be greater than that using eight photographs, and this is reflected in the standard deviations in Tables 3 and 4.

The influences of sex, age and body size on assessment are consistent with existing assumptions. Men tend to underestimate portion size in comparison with women (Table 6), although the size of the percentage error when using eight photographs was small on average (within $\pm 3 \%$ ) for both sexes. The size and range of the errors made by men were substantially greater than those made by women using the average photographs. Analysis by age showed that subjects 65 years and over tended to overestimate in comparison with other age groups (for the eight photographs) or underestimate less (using the average photographs), and this was reflected in the occupational analysis. The striking underestimate of portion size by the heaviest subjects $\left(B M I \geqslant 30 \mathrm{~kg} / \mathrm{m}^{2}\right.$ ) using the eight photographs is consistent with previous observations that heavier subjects tend to understate intake. It may reflect the fact that heavier subjects have larger portions, on average, and are therefore likely to under-report the amount. Curiously, the lightest subjects (BMI $<25 \mathrm{~kg} / \mathrm{m}^{2}$ ) underestimated portion size to the same extent as the heaviest subjects when using the average photographs but not when using the eight photographs.

The use of colour photographs resulted in a mean percentage difference significantly higher than that obtained using black-and-white photographs (Table 7), but the size of the difference between black and white and colour was very small (just over $2 \%$ ). This suggests that well reproduced (i.e. printed not photocopied) black-and-white images are suitable as an aid to portion size estimation (e.g. in postal surveys). Colour photographs* may be more attractive and in a long interview may help to hold a subject's attention.

* An atlas of colour photographs, of eight portion sizes of seventy-six foods, is in preparation for publication in 1995 (M. Nelson, M. Atkinson and J. Meyer, unpublished results). 
There were no significant effects of photograph size on perception. The smallest photograph $(75 \times 100 \mathrm{~mm})$ allowed eight images to be displayed together on one A4 page. This was convenient for purposes of presentation, and facilitated comparison of a set of finely graded differences. The images in the A7 photographs were large enough for the texture of the food to be evident. Smaller images may become merely representational of portion size (i.e. the character or nature of the food is no longer clear), and the authors would suggest that $75 \times 100 \mathrm{~mm}$ should be regarded as the minimum acceptable size for food photographs.

The present evidence points strongly to the value of having a series of photographs rather than single photographs to help subjects estimate portion size. Exactly how many photographs are needed for each food is not known. This and other factors which are likely to influence the subjects' ability to estimate portion size (conceptualization, memory, crockery and cutlery), or the researcher's ability to classify subjects correctly according to level of food consumption or nutrient intake (range of foods for which photographs are available, type of dietary assessment) will be the objective of further studies.

The authors would like to thank James Meyer for the photography, the Ministry of Agriculture, Fisheries and Food for the grant to undertake the work, members of the Nutritional Epidemiology Group Steering Committee who oversaw the design of the project and commented constructively on its analysis, and the volunteers for giving up their time to undertake so diligently what was at times a very tedious task. (Membership of the Nutritional Epidemiology Group Steering Committee: Ms Mary Atkinson (KCL), Miss Alison Black (MRC Dunn Nutrition Unit), Dr Joyce Hughes (MAFF), Mr James Meyer, Ms Alison Mills (MAFF), Dr Michael Nelson (KCL), Dr Richard Shepherd (AFRC Institute of Food Research), Dr Margaret Thorogood (London School of Hygiene and Tropical Medicine). Corresponding members: Dr Annie Anderson (University of Glasgow), Ms Mary Cooper (St Mary's Hospital, Leeds).)

\section{APPENDIX}

Weights $(\mathrm{g})$ of foods at the 5 th and 95 th centiles (photograph numbers 1 and 8 respectively), plus the weights of foods in the intervening six photographs and the interval in weight between photographs; and the weight at the 50th centile (average)

\begin{tabular}{|c|c|c|c|c|c|c|c|c|c|c|}
\hline \multirow[b]{2}{*}{ Food } & \multirow[b]{2}{*}{ Interval } & \multicolumn{8}{|c|}{ Photograph number } & \multirow[b]{2}{*}{ Average } \\
\hline & & 1 & 2 & 3 & 4 & 5 & 6 & 7 & 8 & \\
\hline Mashed potato & $40 \cdot 86$ & 58 & 99 & 140 & 181 & 221 & 262 & 303 & 344 & 159 \\
\hline Boiled potato & $40 \cdot 86$ & 58 & 99 & 140 & 181 & 221 & 262 & 303 & 344 & 159 \\
\hline Quiche & $22 \cdot 28$ & 62 & 84 & 107 & 129 & 151 & 173 & 196 & 218 & 120 \\
\hline Cornflakes & $8 \cdot 71$ & 16 & 25 & 33 & 42 & 51 & 60 & 68 & 77 & 40 \\
\hline Spaghetti & $52 \cdot 85$ & 55 & 108 & 161 & 214 & 266 & 319 & 372 & 425 & 213 \\
\hline Sliced meat & $23 \cdot 14$ & 26 & 49 & 72 & 95 & 119 & 142 & 165 & 188 & 84 \\
\hline
\end{tabular}

\section{REFERENCES}

Byers, T., Marshall, J., Fiedler, R., Zielenzny, M. \& Graham, S. (1985). Assessing nutrient intake with an abbreviated dietary interview. American Journal of Epidemiology 122, 41-50.

Chu, S. Y., Kolonel, L. N., Hankin, J. H. \& Lee, J. (1984). A comparison of frequency and quantitative dietary methods for epidemiologic studies of diet and disease. American Journal of Epidemiology 119, 323-333. 
Edington, J., Thorogood, M., Geekie, M., Ball, M. \& Mann, J. (1989). Assessment of nutritional intake using dietary records with estimated weights. Journal of Human Nutrition and Dietetics 2, 407-414.

Gregory, J., Foster, K., Tyler, M. \& Wiseman, M. (1990). The Dietary and Nutritional Survey of British Adults. London: H.M. Stationery Office.

Guthrie, H. A. (1984). Selection and quantification of typical food portions by young adults. Journal of the American Dietetic Association 12, 1440-1444.

Hankin, J. H., Wilkins, L. R., Kolonel, L. N. \& Yoshizawa, C. N. (1991). Validation of a quantitative diet history method in Hawaii. American Journal of Epidemiology 133, 616-628.

Pietinen, P., Hartman, A. M., Haapa, E., Rasanen, L., Haapakoski, J., Palmgren, J., Albanes, D., Virtamo, J. \& Huttunen, J. K. (1988a). Reproducibility and validity of dietary assessment instruments. I. A self-administered food use questionnaire with a portion size picture booklet. American Journal of Epidemiology 128, 655-666.

Pietinen, P., Hartman, A. M., Haapa, E., Rasanen, L., Haapakoski, J., Palmgren, J., Albanes, D., Virtamo, J. \& Huttunen, J. K. (1988 b). Reproducibility and validity of dietary assessment instruments. II. A qualitative food frequency questionnaire. American Journal of Epidemiology 128, 667-675.

Rutishauser, I. H. E. (1982). Food models, photographs or household measures? Proceedings of the Nutrition Society of Australia 7, 144-145.

Samet, J. M., Humble, C. G., Skipper, B. E. (1984). Alternatives in the collection and analysis of food frequency interview data. American Journal of Epidemiology 120, 572-581.

Tjonneland, A., Overvad, K., Haraldsdottir, J., Bang, S., Ewertz, M. \& Jenson, O. M. (1991). Validation of a semi-quantitative food frequency questionnaire developed in Denmark. International Journal of Epidemiology 20, 906-912. 